\title{
Axisymmetric Slot Perturbation According to Perpendicular Feed for Circularly Polarized Microstrip Antenna
}

\author{
*Seongmin Pyo \\ Department of Information and Communication Engineering \\ Hanbat National University, Daejeon 34158, Rep. of Korea \\ spyo@hanbat.ac.kr
}

\begin{abstract}
This paper presents a new, circularly polarized microstrip antenna based on an axially symmetric $H$-shaped slot perturbation method according to perpendicular-feed network for vehicle to nomadic devices communication system. By means of embedding the slot at the resonator due to the perpendicular feeding structure, the axis-symmetrical geometry of the slot can be obtained. As a result, the loaded axisymmetric slot provides two orthogonal operating TM modes with identical electrical lengths, that is, $T M_{10}$ and $T M_{01}$ modes. Also, the TM 10 and TM 01 modes yield a 90-degree phase differences of the electric field depending on the feeding directions. The proposed antenna successfully demonstrates circularly polarized operations with -10 dB impedance bandwidth of $2.9 \%$, $3 d B$ axial ratio bandwidth of $0.8 \%$, the minimum axial ratio of $0.16 \mathrm{~dB}$ and the antenna gain of $0.52 \mathrm{~dB}$ at the resonant frequency of $2.4 \mathrm{GHz}$.
\end{abstract}

Keywords: microstrip antenna, circular polarization, perpendicular feeding network, $H$-slot perturbation

\section{Introduction}

Since IEEE 802.11p standard was reported in 2010, the antenna has been the key component for modern communication systems, especially, for wireless access in vehicular environments (WAVE) [1-4]. The function of antennas for vehicle to nomadic devices (V2N) communication system is more attractive and challengeable because a vehicular environment requires insensitive multi-path fading and electro-magnetic interferences, which seriously affect system capability and performance degradation. For these reasons, the circular polarization of antennas has become more attractive compared with that of linear polarization due to effectiveness in reliable communication sensitivity and reducing a multipath fading for the orientation between transmitter and receiver in wireless communication [5-7]. Furthermore, to satisfy the demands of the size, weight, cost and ease of system integration, a microstrip antenna is chosen for V2N communication [8-11]. Accordingly, a circularly polarized microstrip antenna can be an excellent candidate to achieve independent signals and to reduce various interferences and in $\mathrm{V} 2 \mathrm{~N}$ communication systems. In previous research, to realize the circular polarization for planar microstrip antennas, various perturbing schemes such as a corner-cut method [12-13], small slit loading [14], asymmetrical defected ground structure [15], and duallooped slit [16] were presented.

In this work, a new and simple perturbing technology for circularly polarized (CP) microstrip antenna without degrading radiation performances is presented for implementing the antenna prototype of V2N communication system. The proposed antenna consists of the axisymmetric slot etched at the square microstrip radiator, and two perpendicular feeding networks. By utilizing the perpendicular feeding network at two sides of the square radiator, orthogonal linear polarizations of the $\mathrm{TM}_{10}$ and $\mathrm{TM}_{01}$ modes can be obtained. Additionally, the axisymmetric slot independently determines the $\mathrm{TM}_{10}$ 
and $\mathrm{TM}_{01}$ frequencies, which supports a 90 degree phase difference between the horizontally and vertically polarized electromagnetic waves. Based on careful investigation of the optimum dimensions, the proposed antenna demonstrates excellent circularly polarized radiation characteristics at the resonant frequency with the minimum axial ratio. Details of operation principle, design, and simulated verifications of the proposed antenna are addressed in following sections.

\section{Antenna Design and Analysis}

Figure 1 shows the configuration of the proposed antenna, which employed two perpendicular feeding networks and the $\mathrm{H}$-shaped slot structure. The $1.6 \mathrm{~mm}$-thick FR4 substrate with the relative dielectric constant of 4.3 is considered for designing an antenna prototype. The proposed antenna consists of the square microstrip patch radiator with the length of $p \mathrm{~mm}$ at the topside and the $\mathrm{H}$-shaped slot, which is etched at the radiator with the length of $l$, the width of $w$, and the gap size of $s \mathrm{~mm}$, respectively. The feeding network is chosen by the directly connected $50-\Omega$ coaxial cable on the bottom side and the 3-dB power divider at the end of coaxial cable on the topside of the substrate. Between the 3-dB power divider and the square microstrip radiator, a quarter-wave impedance transformer to match the antenna impedance is utilized. The directly fed coaxial cable transmission line, which connects the 3 - $\mathrm{dB}$ power divider with two $100-\Omega$ microstrip lines, supplies equally-divided powers to the perpendicular feeding structure.

Figure 2 illustrates the operation principles of the proposed antenna to obtain circularly polarized microstrip antenna based on the axisymmetric slot perturbation depending on the perpendicular feeding network. The RF input powers, which are excited by the coaxial cable transmission line divided equally at the 3 -dB power divider circuitry.

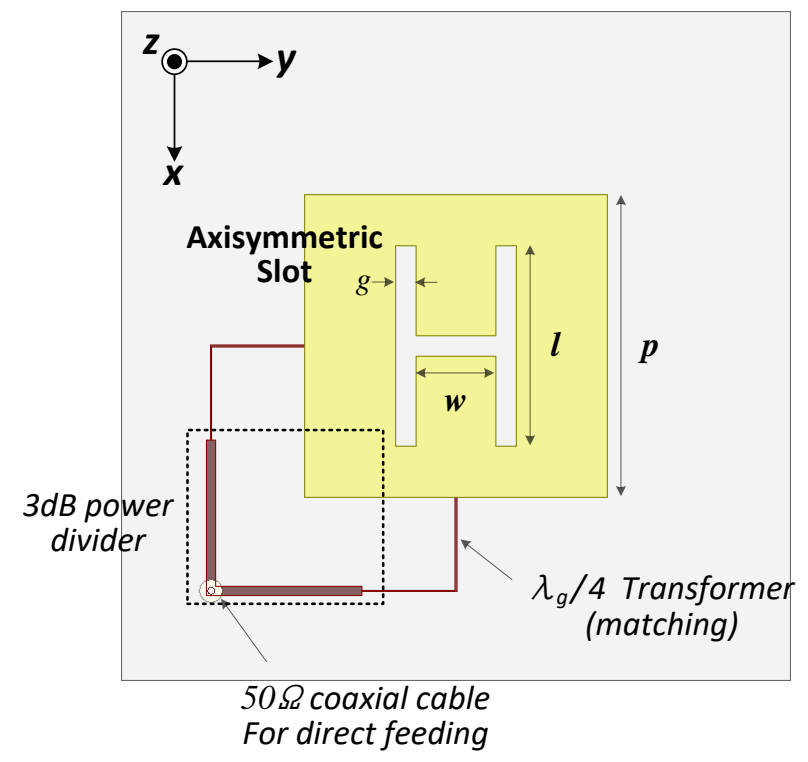

Figure 1. Configuration of the Proposed Antenna Using Axisymmetric Slot According to Orthogonal Feed Network 

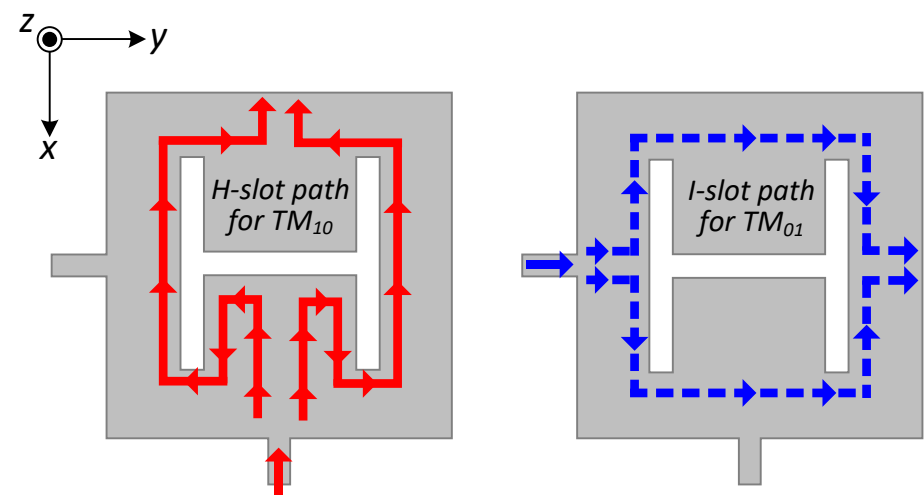

Figure 2. Operation Principle of the Axisymmetric Slot Perturbing Method According to Perpendicular Feeding Direction

The equally divided RF input powers arrive at each side of the radiator after the matching circuit using the quarter-wave impedance transformer. As a result, two perpendicular feeding lines provide the equally dividing incident RF powers with generate vertical and horizontal modes, that is, $\mathrm{TM}_{10}$ and $\mathrm{TM}_{01}$ modes that support linear polarization toward $x$ - and $y$-axis, respectively. Accordingly, the proposed antenna produces two modes due to the perpendicular feeding directions.

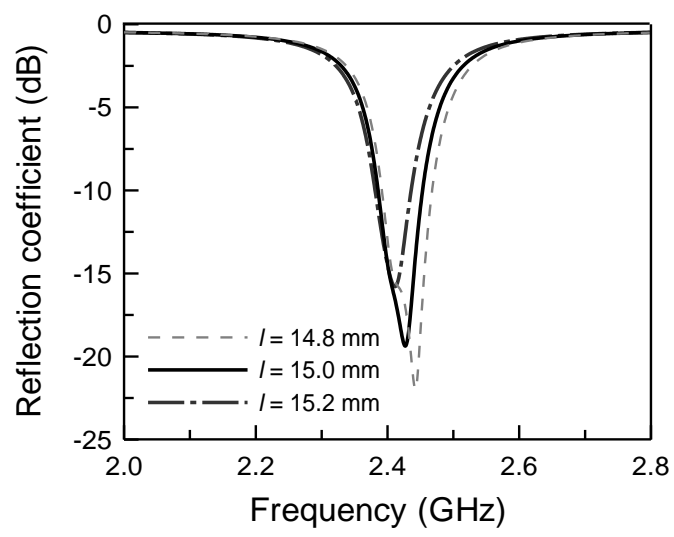

(a)

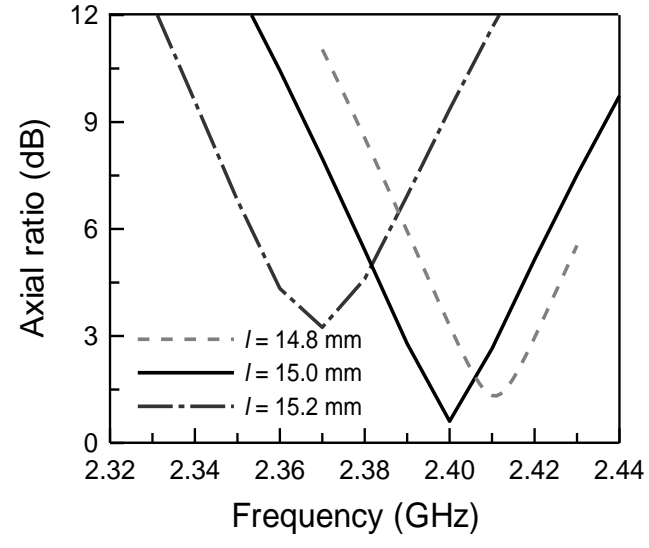

(b)

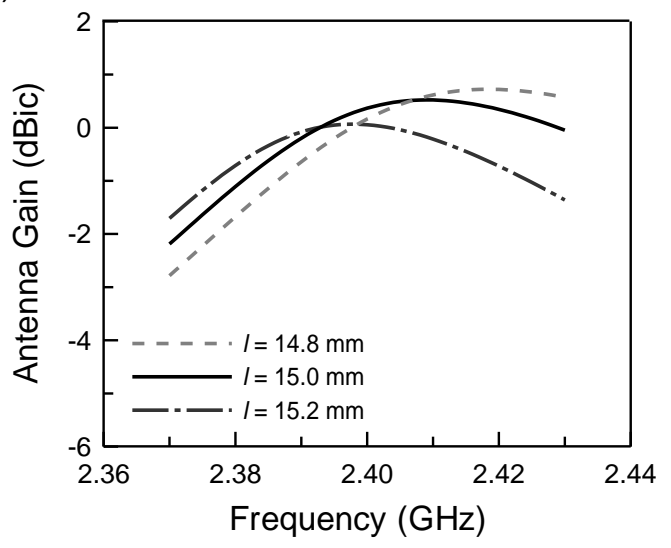

(c)

Figure 3. Simulated Results of the Proposed Antenna Varied with the Parameters of the Slot Length (L). (a) Reflection Coefficient (b) Axial Ratio, and (c) RHCP Antenna Gain 
These $\mathrm{TM}_{10}$ and $\mathrm{TM}_{01}$ frequencies can be independently controlled because the embedded slot is shown as an " $\mathrm{H}$ "-shaped slot geometry for $\mathrm{TM}_{10}$ frequency, and as an "I"-shaped slot geometry for $\mathrm{TM}_{01}$ frequency, respectively. It is noted that the embedded slot acts as the axisymmetric geometry because of the perpendicular feeding directions. Furthermore, these orthogonal modes have identical electric field magnitudes with a 90degree phase difference and show the unique characteristics of controlling independently. Thus, the effect of the embedded slot changed by the feeding positions yields the resonant frequencies for the $\mathrm{TM}_{10}$ and $\mathrm{TM}_{01}$ modes, respectively. In particular, two orthogonal modes to achieve the circular polarization are required to closely separate frequencies. Accordingly, the axisymmetric slot structure is carefully investigated.

Figs. 3 and 4 show the simulated reflection coefficients, axial ratios, and $\mathrm{CP}$ antenna gains based on the parameter study for the axisymmetric slot length of $l$. and the axisymmetric slot width of $w$, respectively. The length and the width of the axisymmetric slot are simulated from $14.8 \mathrm{~mm}$ to $15.2 \mathrm{~mm}$ and from $5.8 \mathrm{~mm}$ to $6.2 \mathrm{~mm}$ for obtaining the resonant frequency of $2.4 \mathrm{GHz}$, respectively. As shown in Figs. 3(a) and 3(b), the upper side frequency of $\mathrm{TM}_{01}$ mode is increased and the frequency with the minimum axial ratio is increased. Also, the magnitudes of the minimum axial ratio are varied when the length of the etched H-shaped slot is increased from $14.8 \mathrm{~mm}$ to $15.2 \mathrm{~mm}$.

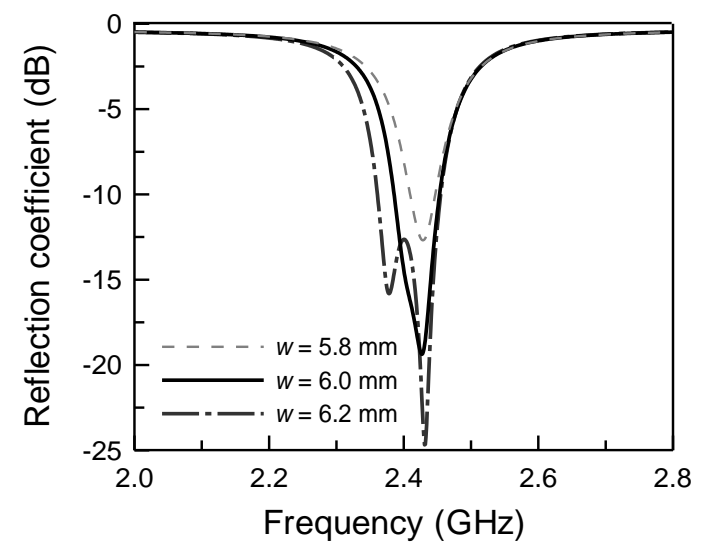

(a)

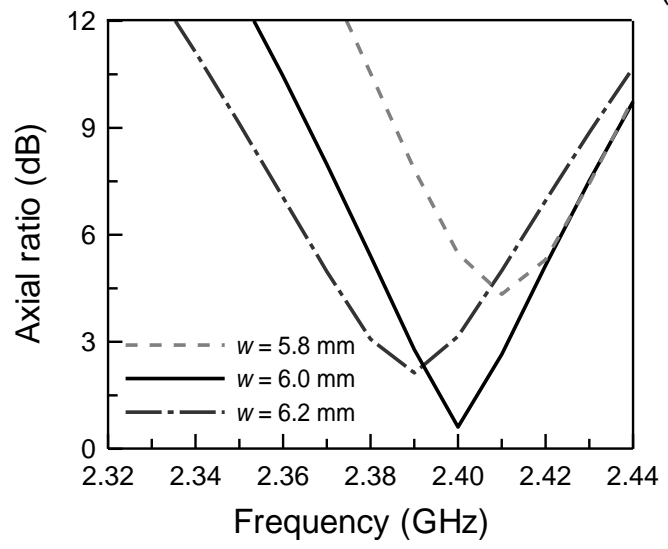

(b)

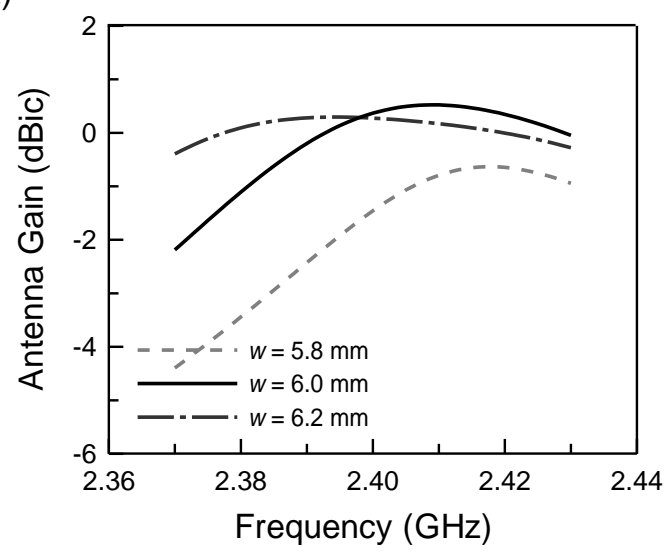

(c)

Figure 4. Simulated Results of the Proposed Antenna Varied with the Parameters, Width of W. (a) Reflection Coefficient (b) Axial Ratio, and (c) RHCP Antenna Gain 


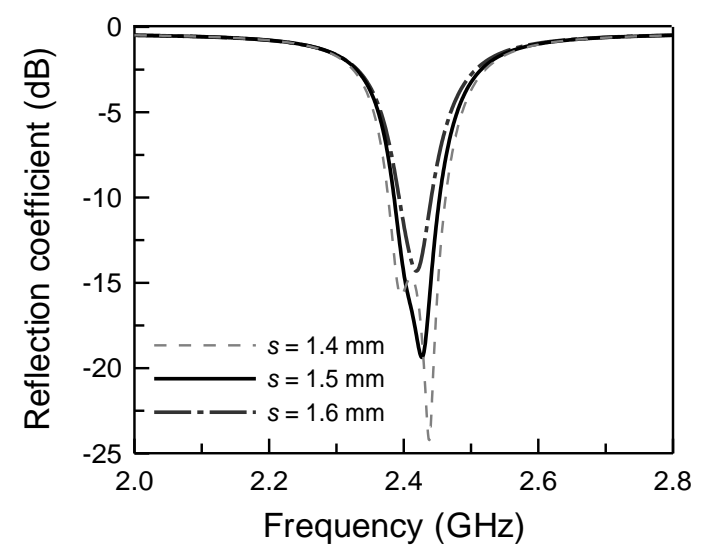

(a)

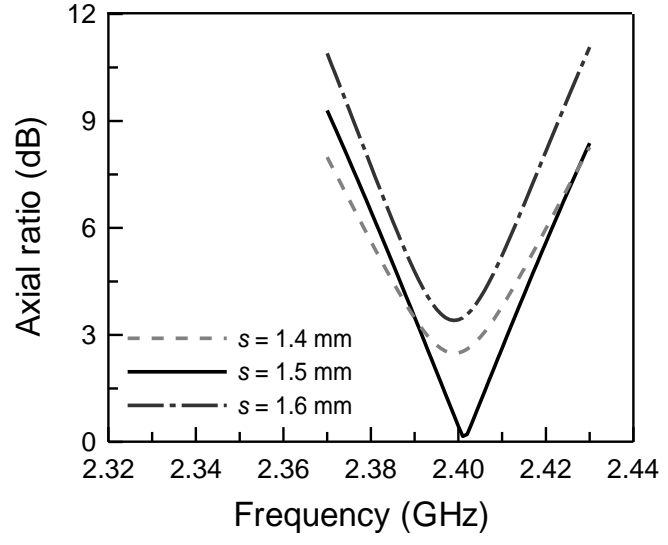

(b)

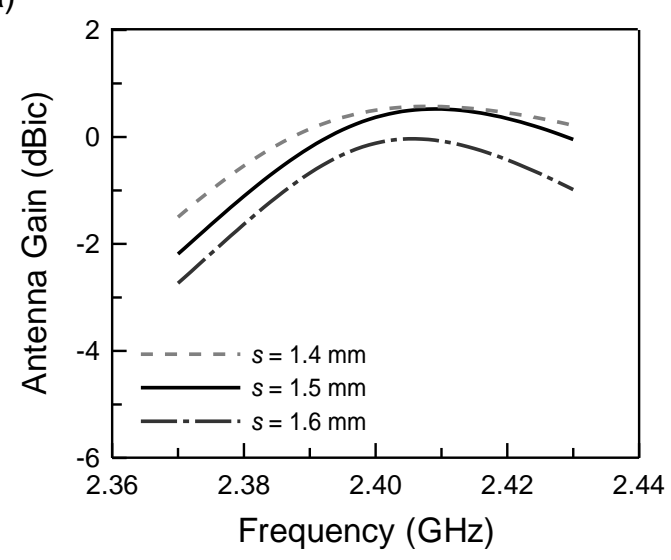

(c)

Figure 5. Simulated Results of the Proposed Antenna Varied with the Parameters, Slot Gap of S. (a) Reflection Coefficient (b) Axial Ratio, and (C) RHCP Antenna Gain

As expected from the operation principle of the proposed antenna as shown in Figure2, the length of the etched slot only controls the electrical length for the $\mathrm{TM}_{01}$ frequency without changing the $\mathrm{TM}_{10}$ frequency. Thus, the upper side of the reflection coefficient moves to the higher frequencies, where the upper frequency of the reflection coefficient is determined by $\mathrm{TM}_{01}$ mode. The lower side of the reflection coefficient is kept because the frequency of $\mathrm{TM}_{10}$ mode is not affected by the length of the etched slot. Because the distance between the frequency of $\mathrm{TM}_{10}$ mode and frequency of $\mathrm{TM}_{01}$ mode is far, the frequency and the magnitude of the axial ratio is increased and varied, respectively. As a result, the frequency with the maximum $\mathrm{CP}$ antenna gain, and the peak gain as shown in Figure 3(c) are increased and varied, respectively. Because the $\mathrm{CP}$ antenna gain is affected by the results of axial ratio, the degenerated axial ratio yields the degenerated $\mathrm{CP}$ antenna gain.

The width of the etched slot is increased from $5.8 \mathrm{~mm}$ to $6.2 \mathrm{~mm}$, the frequency of $\mathrm{TM}_{10}$ is decreased and the frequency of $\mathrm{TM}_{01}$ is sustained as shown in Figure 4(a). It is the opposite case of the length of the axisymmetric slot. The lower side of the reflection coefficients is lowered and the upper side of the reflection coefficient is kept. Similarly, the case of the length of the etched slot, the far distance between the frequency of $\mathrm{TM}_{10}$ mode, and frequency of $\mathrm{TM}_{01}$ mode as shown in Figure 4(b) makes the frequency and the magnitude of the axial ratio decrease and vary, respectively.

As shown in Figure 4(c), because the antenna gain is also affected by the axial ratio, the $\mathrm{CP}$ antenna gain is degenerated as the minimum axial ratio is increased. In other words, the modes for accomplishing the circular polarization of the proposed antenna can 
be independently controlled by the optimized using the width for $\mathrm{TM}_{10}$ and the length for $\mathrm{TM}_{01}$ modes. It is because, in $x$-axis, the width of the etched slot can control the frequency of the $\mathrm{TM}_{10}$, and in $y$-axis, the length of the etched slot can determine the frequency of the $\mathrm{TM}_{01}$. It is a unique characteristic based on the axis-symmetry, which consists of the orthogonal feeding technology with the $\mathrm{H}$-shaped slotted ground structure.

Figure 5 shows the simulated ones based on parameter study for the axisymmetric slot size of $s$. The slot size, that is, gap of $s$, affects both frequencies of the $\mathrm{TM}_{10}$ and $\mathrm{TM}_{01}$ modes. When the gap size is increased from $1.4 \mathrm{~mm}$ to $1.6 \mathrm{~mm}$, the reflection coefficients and axial ratios are deteriorated. The relation between the axial ratio and the RHCP gain is shown as the proportion changes. The slot gap size only makes the magnitude of axial ratio change without shifting resonant frequencies. Accordingly, the increased axial ratios can decrease the lower RHCP antenna gain. From the results as shown in Figures 3, 4, and 5 , the frequencies of the $\mathrm{TM}_{10}$ and $\mathrm{TM}_{01}$ mode can be determined by the axisymmetric slot structure for the length of $l$ and the width of $w$, respectively. In addition, the axisymmetric slot provides a compact radiator size compared with that of without the axisymmetric slot yielded by the slot loading effects.

\section{Results and Discussion}

The physical dimensions of the proposed antenna are optimized based on FEM-based full wave EM simulation to be $p$ of $22.6 \mathrm{~mm}, l$ of $15 \mathrm{~mm}$, and $w$ of $6 \mathrm{~mm}$.

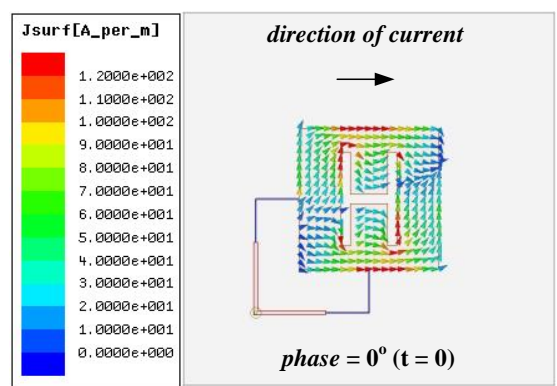

(a)

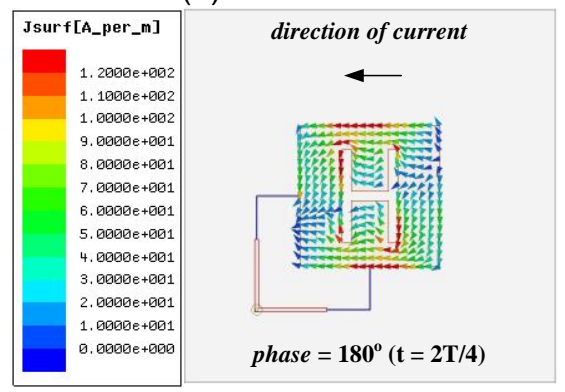

(c)

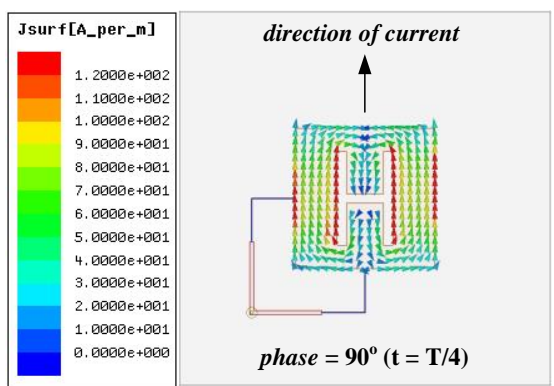

(b)

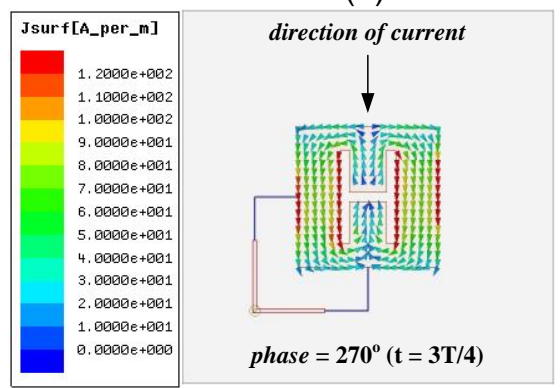

(d)

Figure 6. Computed Electric Surface Current Distributions of the Proposed Antenna at the Resonant Frequency of 2.4 Ghz for (a) Phase of 0 Degree, (b) Phase of 90 Degree, (c) Phase of 180 Degree, and (d) Phase of 270 Degree

The gap of the axisymmetric slot is designed to be $1.5 \mathrm{~mm}$ to obtain optimum righthanded circularly polarized (RHCP) radiation characteristics at $2.4 \mathrm{GHz}$.

Figure 6 shows the simulated electric current distributions of the proposed antenna based on the optimized antenna dimensions. As expected, the surface electrical current distributions are rotated in the counter clockwise direction leading to right-handed circular polarization (RHCP) in the proposed antenna. Because the loaded slot exhibits different 
electrical delays at each feeding point, the orthogonal modes, which are $\mathrm{TM}_{10}$ and $\mathrm{TM}_{01}$ modes, for RHCP operation are utilized.

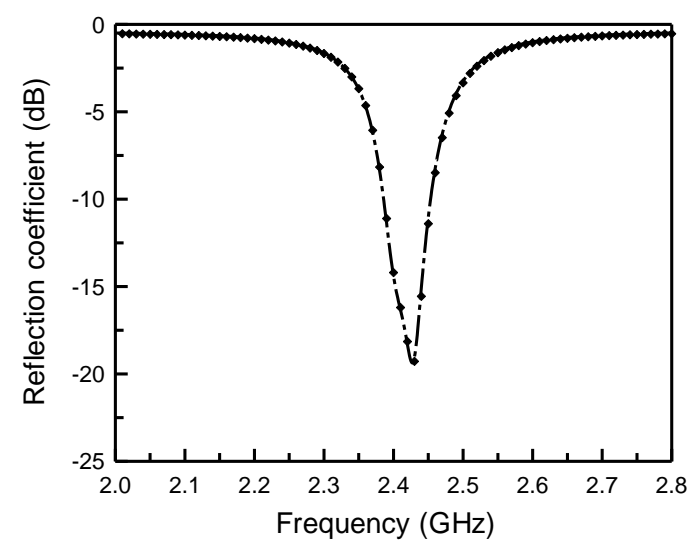

(a)

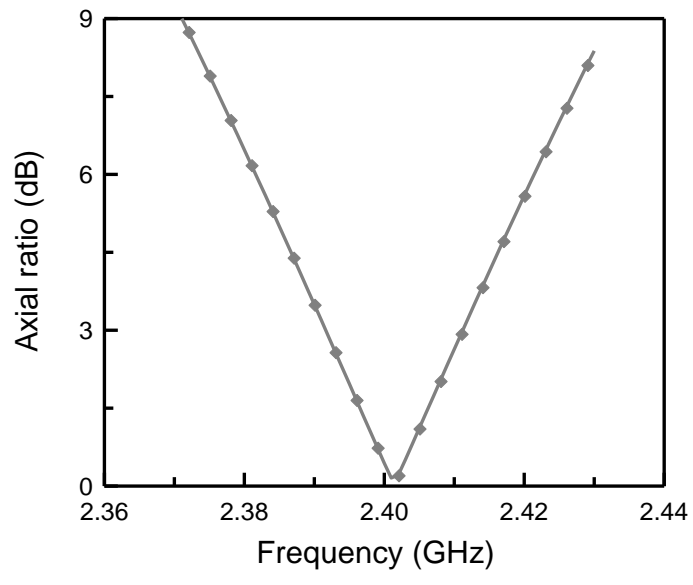

(b)

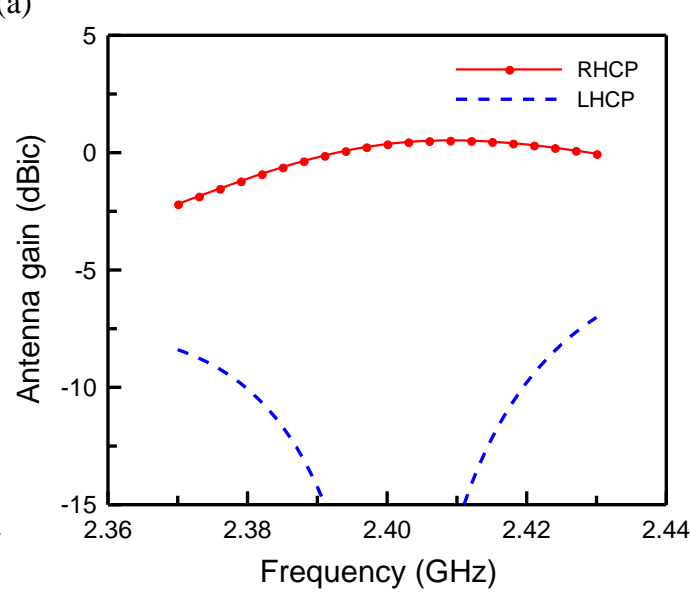

(c)

\section{Figure 7. Characteristics of the Proposed Antenna for (a) Reflection} Coefficient, (b) Axial Ratio, and (c) Antenna Gain

It is noted that the proposed antenna produces the right-handed circular polarization (RHCP). The axisymmetric slot can easily be observed in these figures due to the symmetry in the antenna's geometry with respect to the feed line. Furthermore, if the axisymmetric slot is rotated to 90 degrees, the left-handed circular polarization (LHCP) can be also observed. It is due to the loaded slot geometry being changed by the feeding position.

Figure 7 shows the final optimized results of the reflection coefficient, the axial ratio, and the CP antenna gain of the proposed antenna. In Figure 7(a), the proposed antenna shows the single band operation of $2.4 \mathrm{GHz}$ and the $10-\mathrm{dB}$ bandwidth of $70 \mathrm{MHz}$ from $2.38 \mathrm{GHz}$ to $2.45 \mathrm{GHz}$ with respect to approximately $2.9 \%$ at the center frequency of the $2.415 \mathrm{GHz}$. The minimum reflection coefficients are observed $-19.38 \mathrm{~dB}$ at $2.43 \mathrm{GHz}$. In Figure 7(b), the minimum axial ratio of the proposed antenna is observed by $0.16 \mathrm{~dB}$ at $2.40 \mathrm{GHz}$ and 3-dB axial ratio bandwidth shows $19 \mathrm{MHz}$ from $2.392 \mathrm{GHz}$ to $2.411 \mathrm{GHz}$ with respect to approximately $0.8 \%$ at the center frequency of $2.401 \mathrm{GHz}$, where the resonant frequency of $2.401 \mathrm{GHz}$ with the minimum axial ratio. 


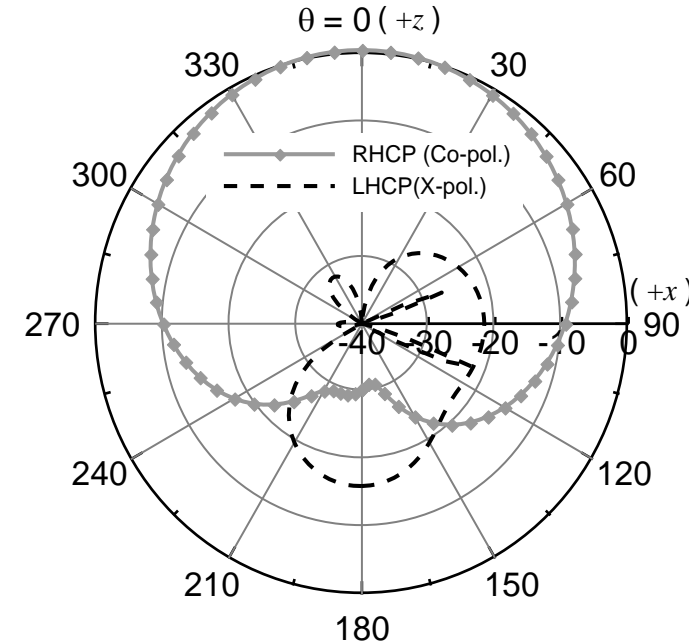

(a)

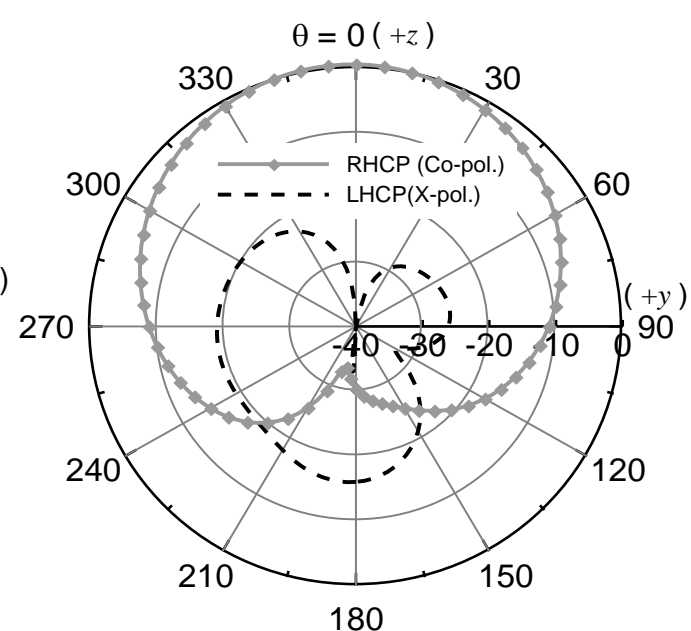

(b)

Figure 8. Circularly Polarized Far-Field Radiation Patterns of the Proposed Antenna at 2.4 Ghz at (a) Zx-Plane and (b) Yz-Plane

In Figure 7(c), the antenna gain of the proposed antenna is obtained by $0.52 \mathrm{dBic}$ at $2.40 \mathrm{GHz}$ for RHCP. The cross-polarized antenna gain, that is, that of LHCP is to be $40.49 \mathrm{dBic}$. It is noted that the cross-polarization ratio shows approximately over $40 \mathrm{~dB}$ at the resonant frequency of $2.40 \mathrm{GHz}$ with the minimum axial ratio of $0.16 \mathrm{~dB}$.

Figure 8 shows the normalized far-field circular polarization radiation patterns of the proposed antenna at the resonant frequency of $2.40 \mathrm{GHz}$ with the minimum axial ratio of $0.16 \mathrm{~dB}$. The results show that broadside radiation patterns with good CP characteristics are obtained at the resonant frequencies in the $z$-direction. The $\mathrm{CP}$ radiation patterns at the resonant frequencies are symmetric; that is, those in the $y z$-plane and the $z x$-plane are identical. The identical CP radiation patterns in $y z$-plane $\left(\phi=90^{\circ}\right.$-plane $)$ and $z x$-plane $(\phi$ $=0^{0}$-plane) are due to the radiator geometry, that is, the square resonator with the H-shape ground slot perturbation and two orthogonal feeding networks. It is shown that the proposed antenna has an excellent circular polarization. The circularly polarized radiation patterns demonstrate excellently symmetrical patterns between $y z$-plane and $z x$-plane with good CP radiation characteristics in the $z$-direction.

\section{Conclusion}

The new design methodology of the electrically small circularly polarized square microstrip antenna was theoretically investigated and discussed in this paper. Based on the geometrically different loading effects of the axisymmetric slot perturbation according to the perpendicular feeding positions, the right-handed circularly polarized antenna was successfully demonstrated. The simulation results show that the proposed antenna exhibited excellent right-handed circularly polarized operation because the electrical phase difference in the axisymmetric slot as a perturbation for each feeding location. In accordance with, the proposed antenna may be an excellent candidate for modern mobile communication systems such as WLAN, satellite communication, and the like, and the proposed antenna can also improve wireless communication capability and performances for future V2N system. 


\section{Acknowledgments}

This research was supported by Basic Science Research Program through the National Research Foundation of Korea (NRF) funded by the Ministry of Science, ICT \& Future Planning (2014R1A1A1004241).

\section{References}

[1] Part 11: Wireless LAN Medium Access Control (MAC) and Physical Layer (PHY) specifications: Amendment 7: Wireless Access in Vehicular Environment (WAVE), IEEE Standard 802, July 2010.

[2] H. Hartenstein and K. Laberteaux, "VANET: Vehicular Applications and Inter-Networking Technologies", John Wiley and Sons, (2010).

[3] W. Xiang, J. Gozalvez, Z. Niu, O. Altintas, and E. Ekici, "Wireless Access in Vehicular Environments", EURASIP Journal on Wireless Communications and Networking, vol. 2009, paper ID. 576217, pp. 2

[4] S. Eichler, "Performance Evaluation of the IEEE 802.11p WAVE Communication Standard", Proc. of IEEE 66th Vehicular Technology Conference, Baltimore, Maryland USA, (2007) 30 September-03 October.

[5] F.-S. Chang, K.-L. Wong, and T.-W. Chiou, "Low-cost broadband circularly polarized patch antenna", IEEE Transactions on Antennas and Propagation, vol. 51, no. 10, (2003), pp. 3006-3009.

[6] J. R. James, P. S. Hall, and C. Wood, "Microstrip Antenna Theory and Design", Peter Peregrinus, London, (1981).

[7] R. Garg, P. Bhartia, I. Bahl, and A. Ittipiboon, "Microstrip Antenna Design Handbook", Artech House, (1995).

[8] S. Pradhan, S.-K. Noh and D.-Y. Choi, "Design of Inset Microstrip Patch Antenna for Wireless Power Transmission at $2.45 \mathrm{GHz}$ ", Journal of Information and Communication Convergence Engineering, vol. 10, no. 2, (2012), pp. 123-128.

[9] S. Pyo and Y. Sung, "Microstrip Antenna Perturbed by a Defected Ground Structure with a Reconfigurable Polarization Capability", Microwave and Optical Technology Letters, vol. 54, no. 1, (2012), pp. 58-61.

[10] J.-H. Yoon, "Circular Ring Open-Ended Monopole Antenna with Strip for WLAN Dual-Band Operations", Journal of Information and Communication Convergence Engineering, vol. 12, no. 1, (2014), pp. 1-7.

[11] S. Pyo and Y. Sung, "A Circularly Polarized Microstrip Antenna with an Arrow-Shaped Slotted Ground", Microwave and Optical Technology Letters, vol. 54, no. 1, (2012), pp. 271-273.

[12] R. Bancroft, "Microstrip and Printed Antenna Design", Scitech, Raleigh, NC, (2006).

[13] Y. Sung, T. U. Jang, and Y.-S. Kim, "Reconfigurable Microstrip Patch Antenna for Switchable Polarization," IEEE Microwave and Wireless Components Letters, vol. 14, no. 11, (2004), pp. 534-536.

[14] Y. Sung, "Polarization Diversity Antenna with L-Type Slit," IEEE Transactions on Antennas and Propagation, vol. 56, no. 9, (2008), pp. 3053-3054.

[15] S. Pyo and Y. Sung, "A Circularly Polarized Microstrip Antenna with an Arrow Shaped Slotted Ground," Microwave and Optical Technology Letters, vol. 54, no. 1, (2012), pp. 271-273.

[16] S. Pyo, "Switchable Circularly-Polarised Square Ring Antenna Controlled by Dual-Loaded Dual-Loop," Electronics Letters, vol. 50, no. 6, (2014), pp. 428-429.

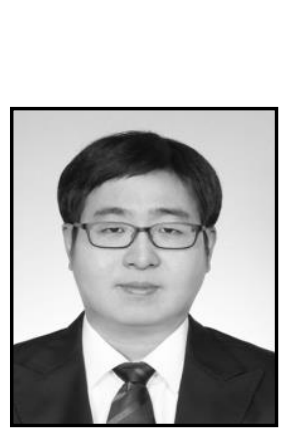

\section{Author}

Seongmin Pyo, received his B.Eng., M. Eng., and Ph.D. degrees from Korea University, Seoul, Korea, in 2002, 2004, and 2011, respectively. In 2011, he was a Senior Researcher with Agency for Defense Development (ADD), where he wrote a number of research about the ISR-UAV communication system with adaptive digital monopulse trackers for national security. In February 2013, he joined the faculty of Hanbat National University, where he is currently an Assistant Professor. His research interests include metamaterial-based electromagnetic theories, reconfigurable antennas, RF \& microwave components, and systems. 
International Journal of Control and Automation

Vol. 10, No. 5 (2017) 